\title{
Mathematica Applications on Time Scales
}

\author{
Ahmet Yantır and Ünal Ufuktepe \\ Izmir Institute of Technology, Department of Mathematics, \\ Urla, Izmir, Turkey \\ \{ahmetyantir, unalufuktepe\}@iyte.edu.tr
}

\begin{abstract}
Stefan Hilger introduced the calculus on time scales in order to unify continuous and discrete analysis in 1988. The study of dynamic equations is an active area of research since time scales unifies both discrete and continuous processes, besides many others. In this paper we give many examples on derivative and integration on time scales calculus with Mathematica. We conclude with solving the first order linear dynamic equation $N^{\Delta}(t)=N(t)$, and show that the solution is a generalized exponential function with Mathematica.
\end{abstract}

\section{Introduction}

Stefan Hilger introduced the calculus on time scales in order to unify continuous and discrete analysis in 1988. Some works in this direction are $[1,3,6,7]$. There are many applications on time scales in [2].

In this paper, first we give the definitions of time scale, forward and backward jump operators which are the required tools of time scale calculus. Time scale calculus as calculus of integers can be examined in two parts: $\Delta$-derivative and $\Delta$-integration, $\nabla$-derivative and $\nabla$-integration. In this study we only deal with $\Delta$ - derivative and $\Delta$-integration. We give some applications on $\Delta$-derivative and $\Delta$-integration with Mathematica. We create TimeScale package to calculate the backward and forward jump operators and derivative of the functions defined a time scale. We give the Mathematica codes for $\Delta$-integration. Similarly, $\nabla$ integration can be done with Mathematica. We also give a brief introduction to linear dynamic equation and solve an application due to [2].

\section{Basic Definitions and Mathematica Codes}

A time scale (measure chain) $\mathbf{T}$, is a nonempty, closed subset of real numbers. The examples of time scales defined with Mathematica can be found in $[9,10]$.

Let $\mathbf{T}$ be a time scale. The forward jump operator $\sigma: \mathbf{T} \rightarrow \mathbf{T}$ and the backward jump operator $\rho: \mathbf{T} \rightarrow \mathbf{T}$ are defined by $\sigma(t)=\inf \{s: s \in \mathbf{T}, s>t\}$ and $\rho(t)=\sup \{s: s \in \mathbf{T}, s<t\}$ for all $t \in \mathbf{T}$ respectively.

If $\mathbf{T}=\mathbf{R}$, then $\sigma(t)=t$, and $\rho(t)=t$ and if $\mathbf{T}=\mathbf{Z}$, then $\sigma(t)=t+1$, and $\rho(t)=t-1$. Also $\sigma(\max \mathbf{T})=\max \mathbf{T}$ and $\rho(\min \mathbf{T})=\min \mathbf{T}$. 
$t$ is called right dense if $\sigma(t)=t$, left dense if $\rho(t)=t$ and right scattered if $\sigma(t)>t$, left scattered if $\rho(t)<t$. If $t$ is both left and right dense then $t$ is called dense point, $t$ is isolated point if it is both left and right scattered. The graininess function $\mu: \mathbf{T} \rightarrow[0, \infty)$ is defined by $\mu(t)=\sigma(t)-t$.

We define the forward operator in two different ways, one for purely discrete sets and the other one for combination of discrete points and closed intervals as follows:

\section{$\operatorname{In}[1]:=<<$ TimeScale \\ $\operatorname{In}[2]:=\operatorname{sigma}[4, \mathrm{i} / 2]$}

In this command the first component stands for the point, and the second component stands for the rule of time scale. The values of $\sigma$ for the same point are different for different time scales:

\section{$\operatorname{In}[3]:=\operatorname{sigma}\left[4, i^{2}\right]$}

In order to remove the deficiency we form another command to compute the value of $\sigma$ for more general time scales as follows:

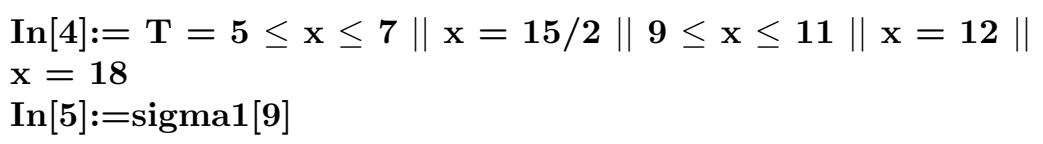

Similarly, we can compute backward jump operator, the dual of forward jump operator, with as follows:

$$
\begin{aligned}
& \operatorname{In}[6]:=\operatorname{rho}\left[4, i^{2}\right] \\
& \operatorname{In}[7]:=\operatorname{rho}\left[\frac{15}{2}\right]
\end{aligned}
$$

\section{Derivative on Time Scales}

In order to define the derivative on time scale we need the following set $\mathbf{T}^{k}$ which is derived from the time scale $\mathbf{T}$ as follows:

$$
\mathbf{T}^{k}= \begin{cases}\mathbf{T}-\{\max \mathbf{T}\} & \text { if } \max \mathbf{T}<\infty \text { and } \max \mathbf{T} \text { is right scattered } \\ \mathbf{T}, & \text { otherwise. }\end{cases}
$$

Definition 1. Let $f: \boldsymbol{T} \rightarrow \boldsymbol{R}$ and $t \in \boldsymbol{T}^{k}$. If there exists a neighborhood $U_{t}$ such that

$$
|f(\sigma(t))-f(s)-a[\sigma(t)-s]| \leq \epsilon|\sigma(t)-s|
$$

is satisfied for all $t, a \in \boldsymbol{R}$ and $s \in U_{t}$. Then $f$ is $\Delta$-differentiable at the point $t$ and $a$ is called $\Delta$-derivative of $f$ at the point $t$.

$$
a=f^{\Delta}(t)=\left\{\begin{array}{cl}
\lim _{s \rightarrow t} \frac{f(\sigma(t))-f(s)}{\sigma(t)-s}, & \text { if } \mu(t)=0 ; \\
\frac{f(\sigma(t))-f(t)}{\mu(t)}, & \text { if } \mu(t)>0 .
\end{array}\right.
$$

$\Delta$-derivative is defined on $\mathbf{T}^{k}=\mathbf{T}-\{\max \mathbf{T}\}$, not on the whole time scale. If $t=\max \mathbf{T}$ then a neighborhood $U_{t}$ of $t$ contains only $t$. So the inequality (1) can be written only for $s=t$, 


$$
\begin{aligned}
|f(\sigma(t))-f(t)-a[\sigma(t)-t]| & \leq \epsilon|\sigma(t)-t| \\
|f(t)-f(t)-a[t-t]| & \leq \epsilon|t-t| \\
0 & \leq 0
\end{aligned}
$$

Thus the definition of $\Delta$-derivative is satisfied for every value of $a$. Then $a$ can not be determined uniquely.

Let $f: \mathbf{T} \rightarrow \mathbf{R}$ and $t \in \mathbf{T}^{k}$. The following two results can be obtained from formula (2):

If $f$ is continuous at $t$ and $t$ is right scattered then $\mathrm{f}$ has a $\Delta$-derivative at $t$ and

$$
f^{\Delta}(t)=\frac{f(\sigma(t))-f(t)}{\mu(t)} .
$$

If $t$ is right dense then $\mathrm{f}$ has a $\Delta$-derivative at $t$ if and only if

$$
\lim _{s \rightarrow t} \frac{f(t)-f(s)}{t-s}
$$

is finite. Then

$$
f^{\Delta}(t)=\lim _{s \rightarrow t} \frac{f(t)-f(s)}{t-s} .
$$

If we unify the above results we get the useful formula, summarizing the $\Delta$ derivative:

$$
f(\sigma(t))=f(t)+f^{\Delta}(t) \mu(t) .
$$

We can formulate the properties of $\Delta$ derivative of two $\Delta$ differentiable functions such as linearity, the product rule, and the quotient rule as follows:

$$
\begin{gathered}
(f+g)^{\Delta}(t)=f^{\Delta}(t)+g^{\Delta}(t) \\
(f g)^{\Delta}(t)=f^{\Delta}(t) g(t)+f(\sigma(t)) g^{\Delta}(t)=f(t) g^{\Delta}(t)+f^{\Delta}(t) g(\sigma(t)) . \\
\left(\frac{f}{g}\right)^{\Delta}(t)=\frac{f^{\Delta}(t) g(t)-f(t) g^{\Delta}(t)}{g(t) g(\sigma(t))} .
\end{gathered}
$$

Clearly $(1)^{\Delta}=0$ and $(t)^{\Delta}=1$ from (2). So by using formula (7) we can find $\left(t^{2}\right)^{\Delta}=(t \cdot t)^{\Delta}=t+\sigma(t)$. We can use (8) to compute

$$
f^{\Delta}(t)=\frac{-2(t+\sigma(t))}{\left(t^{2}-1\right)\left((\sigma(t))^{2}-1\right)}
$$

where $f(t)=\frac{t^{2}+1}{t^{2}-1}$.

For $\mathbf{T}=\mathbf{N}_{0}^{\frac{1}{2}}=\left\{\sqrt{n}: n \in \mathbf{N}_{0}\right\}$, if $t \in \mathbf{T}$ then $\sigma(t)=\sqrt{t^{2}+1}$. Thus $f^{\Delta}(t)=\frac{-2\left(t+\sqrt{t^{2}+1}\right)}{\left(t^{2}-1\right) t^{2}}$.

For $\mathbf{T}=\left\{\frac{n}{2}: n \in \mathbf{N}_{0}\right\}$, if $t \in \mathbf{T}$ then and $\sigma(t)=\frac{2 t+1}{2}$. Thus $f^{\Delta}(t)=-\frac{4 t+1}{t^{2}+t-\frac{3}{4}}$.

Further results and applications are included in $[2,4]$. 
We define this new derivative operator with Mathematica in two different ways, one for time scales which are the combination of discrete and closed intervals and the other for time scales including only isolated points as follows:

$$
\begin{aligned}
& \operatorname{In}[8]:=\text { Clear[sigma, } T] \\
& \operatorname{In}[\mathbf{9}]:=\mathrm{f}\left[\mathrm{t}_{-}\right]:=\frac{t^{2}+1}{t^{2}-1} \\
& \operatorname{In}[\mathbf{1 0}]:=\mathbf{T}=\mathbf{5} \leq \mathrm{x} \leq \mathbf{7}\left\|\mathrm{x}=\frac{\mathbf{1 5}}{\mathbf{2}}\right\| \mathbf{9} \leq \mathrm{x} \leq \mathbf{1 1}\|\mathrm{x}=\mathbf{1 2}\| \mathrm{x}=\mathbf{1 8} \\
& \operatorname{In}[11]:=\text { Tderivative1[f, } 7]
\end{aligned}
$$

Tderivative 2 determines the derivative of a function on a discrete time scale.

$\operatorname{In}[12]:=$ Clear[f, $\mathbf{T}, \mathbf{n}, \mathbf{t}$, sigma, i, deriv]

$\operatorname{In}[13]:=$ Tderivative $2\left[t^{3}, 4,2^{i}\right]$

\section{Integration on Time Scale}

Definition 2. A function $f: \boldsymbol{T} \rightarrow \boldsymbol{R}$ is called regulated if it has finite rightsided limits at all right dense points in $\boldsymbol{T}$ and it has finite left-sided limits at all left dense points in $\boldsymbol{T}$.

A function $f: \mathbf{T} \rightarrow \mathbf{R}$ is called rd-continuous if it is continuous at all right-dense points in $\mathbf{T}$ and its left-sided limits exist (finite) at all left-dense points in $\mathbf{T}$. The set of rd-continuous functions in $\mathbf{T}$ is denoted by $C_{r d}$.

Theorem 1. (Existence of antiderivative) Every rd-continuous function has an antiderivative. In particular if $t_{0} \in \boldsymbol{T}$, then $F$ defined by

$$
F(t):=\int_{t_{0}}^{t} f(\tau) \Delta \tau \quad \text { for } \quad t \in \boldsymbol{T}
$$

is an antiderivative of $f$.

Proof (4).

Theorem 2. If $f \in C_{r d}$ and $t \in \boldsymbol{T}^{k}$, then

$$
\int_{t}^{\sigma(t)} f(\tau) \Delta \tau=(\sigma(t)-t) \mu(t) f(t) .
$$

Proof (4).

Theorem 3. Let $a, b \in \boldsymbol{T}$ and $f \in C_{r d}$. If $[a, b]$ consists of only isolated points, then

$$
\int_{a}^{b} f(t) \Delta t= \begin{cases}\sum_{t \in[a, b)} \mu(t) f(t), & a<b ; \\ 0 & a=b ; \\ -\sum_{t \in[b, a)} \mu(t) f(t), & a>b .\end{cases}
$$


Proof. Assume that $a<b$ and let $[a, b]=\left\{t_{0}, t_{1}, t_{2}, \ldots, t_{n}\right\}$ where

$$
\begin{aligned}
& a=t_{0}<t_{1}<t_{2}<\ldots<t_{n}=b . \\
\int_{a}^{b} f(t) \Delta t= & \int_{t_{0}}^{t_{1}} f(t) \Delta t+\int_{t_{1}}^{t_{2}} f(t) \Delta t+\ldots+\int_{t_{n-1}}^{t_{n}} f(t) \Delta t \\
= & \sum_{i=0}^{n-1} \int_{t_{i}}^{t_{i+1}} f(t) \Delta t \\
= & \sum_{i=0}^{n-1} \int_{t_{i}}^{\sigma\left(t_{i}\right)} f(t) \Delta t \\
= & \sum_{i=0}^{n-1}\left(\sigma\left(t_{i}\right)-t_{i}\right) f\left(t_{i}\right) \\
= & \sum_{t \in[a, b)}(\sigma(t)-t) f(t)
\end{aligned}
$$

If $a>b$, by using the fact

$$
\int_{a}^{b} f(t) \Delta t=-\int_{b}^{a} f(t) \Delta t
$$

we obtain

$$
\int_{a}^{b} f(t) \Delta t=-\sum_{t \in[b, a)}(\sigma(t)-t) f(t)
$$

which is the desired result.

If $f$ is defined on a time scale which consists of only isolated points then by using Theorem 3 it is possible to evaluate the integral as follows:

$\operatorname{In}[14]:=$ Clear $[\mathrm{f}, \mathrm{g}, \mathrm{l}, \mathrm{u}]$;

$\operatorname{In}[15]:=$ Tintegrate1 $\left[\mathrm{f}_{-}, \mathrm{g}_{-}, \mathrm{l}_{-}, \mathrm{u}_{-}\right]:=\operatorname{Module}[\{\mathrm{T}, \mathrm{s}\}$, $\mathbf{T}=$ Table $[\mathrm{g},\{\mathbf{i}, \mathbf{l}, \mathbf{u}\}]$;

Print [" The time scale is $=$ ", $\mathrm{T}]$;

$\mathbf{s}=\operatorname{Sum}[(\mathbf{f} / . \mathbf{x}->\mathbf{T}[[\mathbf{i}]]) *(\mathbf{T}[[\mathbf{i}+\mathbf{1}]]-\mathbf{T}[[\mathbf{i}]]),\{\mathbf{i}, \mathbf{1}, \mathbf{u}-\mathbf{l}\}] ;$

Print [" The value of the integral $=$ ", s]]

In[16]:= Tintegrate1 $\left[x^{3}, \mathrm{i} / \mathbf{2}, \mathbf{1}, \mathbf{1 0}\right]$

Out $[16]:=$ The time scale is $=\left\{\frac{1}{2}, 1, \frac{3}{2}, 2, \frac{5}{2}, 3, \frac{7}{2}, 4, \frac{9}{2}, 5\right\}$

The value of the integral $=\frac{2025}{16}$

In this module $f$ denotes the integrant, $g$ denotes the rule of discrete time scale in terms of $i, l$ denotes the lower bound, and $u$ denotes the upper bound of the integral. 
If $f$ is defined on an arbitrary time scale which is the combination of closed intervals and discrete sets then we can evaluate the value of the integral:

$\operatorname{In}[17]:=A=\operatorname{Interval}[\{1,3\},\{5,5\},\{6,8\},\{12,13\}]$

$\operatorname{In}[18]:=$ Tintegrate2 $\left[\mathrm{f}_{-}, \mathrm{a}_{-}, \mathrm{b}_{-}\right]:=\operatorname{Module}[\{\mathrm{B}, \mathrm{i}, \mathrm{j}, \mathrm{Sum} 1, \mathrm{Sum} 2\}$, $\mathbf{B}=$ IntervalIntersection $[\mathrm{A}, \operatorname{Interval}[\{\mathrm{a}, \mathrm{b}\}]] ; \mathrm{i}=\mathbf{1}$;

Print [" The time scale is = ",A];

Print [" The Integral Interval $=$ " ,B];

While $[B[[i]][[2]] \neq \operatorname{Max}[B], i=i+1] ; \operatorname{Sum} 1=0 ; \operatorname{Sum} 2=0$;

$\mathbf{j}=1$; While $[\mathbf{j} \leq \mathbf{i}$, Sum1 $=\operatorname{Sum} 1+\operatorname{Integrate}[\mathbf{f},\{\mathrm{x}, \mathrm{B}[[\mathbf{j}]][[1]], \mathrm{B}[[\mathbf{j}]][[2]]\}]$;

Print[" Ordinary integration from ",B $[[\mathbf{j}]][[1]]$, " to " ,B $[[\mathbf{j}]][[2]]]$;

$\mathrm{j}++;]$; Print [" The value of ordinary integral=",Sum1]; $\mathrm{j}=1$;

While $[\mathbf{j}<\mathbf{i}$, Sum2 $=$ Sum2 $+(\mathbf{f} / . x->B[[j]][[2]]) *(B[[j+1]][[1]]-B[[j]][[2]])$;

Print[" Discrete integral from $=$ ",B $[[\mathrm{j}]][[2]], "$ to ",B $[[\mathrm{j}+1]][[1]]]$;

$\mathrm{j}++;$; Print [" The value of discrete integral=",Sum2];

Print[" The value of Time Scale integral=", Sum1+ Sum2 ];

$\operatorname{In}[19]:=$ Tintegrate2 $\left[x^{2}, 2,7\right] / / \mathbf{N}$

Here $f$ denotes the integrant, $a$ denotes the lower bound, and $b$ denotes the upper bound of the integral.

\section{Linear Dynamic Equations and Applications}

In this section we try to solve first order linear dynamic equations with Mathematica.

Definition 3. A function $p: \boldsymbol{T} \rightarrow \boldsymbol{R}$ is called regressive if $1+\mu(t) p(t) \neq 0$ is satisfied for all $t \in \boldsymbol{T}$.

Concerning the initial value problems

$$
y^{\Delta}=p(t) y, \quad y\left(t_{0}\right)=1
$$

Hilger [6] proved the existence and uniqueness of (9) under the conditions $p$ is rd-continuous and regressive. And (9) is called regressive if $p$ is regressive.

The solution of (9) is called the exponential function and denoted by $e_{p}\left(\cdot, t_{0}\right)$. The explicit formula for $e_{p}(t, s)$ is given by

$$
e_{p}(t, s)=\exp \left\{\int_{s}^{t} \xi_{\mu(\tau)}(p(\tau)) \Delta \tau\right\}
$$

where $\xi_{h}(z)$, the cylindrical transformation,

$$
\xi_{h}(z)=\left\{\begin{array}{cl}
\frac{\log (1+h z)}{h}, & \text { if } h \neq 0 \\
z, & \text { if } h=0 .
\end{array}\right.
$$


The properties of the exponential functions are given by Bohner and Peterson [4] and summarized in [3].

By using the exponential function the solutions of many dynamic equations can be found in a similar way as in ordinary differential equations. As an example by considering the dynamic equation

$$
y^{\Delta^{3}}-2 y^{\Delta^{2}}+y^{\Delta}+2 y=0
$$

where $y^{\Delta^{i}}, i=2,3$ denotes the $i^{\text {th }}$ delta derivative of $y$ and assuming that $y(t)=e_{\lambda}\left(t, t_{0}\right)$ with constant $\lambda$ is the solution, we can find that the linear combination of $e_{-1}\left(t, t_{0}\right), e_{1}\left(t, t_{0}\right), e_{2}\left(t, t_{0}\right)$ is the solution of the dynamic equation above.

As another example we consider the initial value problem

$$
y^{\Delta^{2}}=a^{2} y, y\left(t_{0}\right)=1, y^{\Delta}\left(t_{0}\right)=0
$$

where $a$ is a regressive constant. By using the same technique we find that both $e_{a}\left(t, t_{0}\right), e_{-a}\left(t, t_{0}\right)$ and therefore $\alpha e_{a}\left(t, t_{0}\right)+\beta e_{-a}\left(t, t_{0}\right)$ solve the dynamic equation. By using initial conditions we find $\alpha=\beta=\frac{1}{2}$. Thus

$$
y(t)=\frac{e_{a}\left(t, t_{0}\right)+e_{-a}\left(t, t_{0}\right)}{2}
$$

is the solution.

If $p$ and $-p$ are regressive and rd-continuous then the hyperbolic functions are defined by

$$
\cosh _{p}=\frac{e_{p}+e_{-p}}{2}, \sinh _{p}=\frac{e_{p}-e_{-p}}{2} .
$$

Similarly the trigonometric functions are defined by the means of the solution of the initial value problem

$$
y^{\Delta^{2}}=-a^{2} y, y\left(t_{0}\right)=1, y^{\Delta}\left(t_{0}\right)=0 .
$$

The following example is due to Agarwal, Bohner, O'Regan and Peterson [3] and is an application of linear dynamic equation.

Example 1. Let $N(t)$ be the amount of plants of one particular kind at time $\mathrm{t}$ in a certain area. By experiments we know that according to $N^{\prime}=N$ during the months of April until September. At the beginning of the October, all plants suddenly die, but seeds remain in the ground and start growing again at the beginning of April with $\mathrm{N}$ being doubled. We model this situation using the time scale

$$
\mathbf{T}=\bigcup_{k=0}^{\infty}[2 k, 2 k+1]
$$

where $t=0$ is April 1 of the current year, $t=1$ is the October 1 of the current year, $t=2$ is the April 1 of the next year, $t=3$ is October 1 of the next year, and so on. We have

$$
\mu(t)=\left\{\begin{array}{l}
0, \text { if } 2 k \leq t<2 k+1 \\
1, \text { if } t=2 k+1
\end{array}\right.
$$


On $[2 k, 2 k+1)$ we have $N^{\prime}=N$, i.e. $N^{\Delta}=N$. However we also know that $N(2 k+2)=2 N(k+1)$, i.e. $N(2 k+1)=N(2 k+1)$, i.e. $N^{\Delta}=N$ at $2 k+1$. As a result $\mathrm{N}$ is the solution of the dynamic equation $N^{\Delta}=N$. Thus, if $\mathrm{N}(0)=1$ is given, $\mathrm{N}$ is exactly $e_{1}(t, 0)$ on the time scale.

In order to solve this problem we give the following Mathematica codes:

$\operatorname{In}[20]:=\operatorname{int}\left[i_{-}\right]:=\operatorname{Interval}\left[\left\{2 * i_{,} *^{*}+1\right\}\right] ;$

$\operatorname{In}[21]:=\mathbf{A}=$ IntervalUnion [int[0], $\operatorname{int}[1], \operatorname{int}[2], \operatorname{int}[3], \operatorname{int}[4], \operatorname{int}[5]$, $\operatorname{int}[6], \operatorname{int}[7], \operatorname{int}[8], \operatorname{int}[9]] ;$

$\operatorname{In}[22]:=$ DynamicSolve $\left[p_{-}, a_{-}, b_{-}\right]:=\operatorname{Module}[\{B, i, j, \operatorname{Sum} 1$,

Sum2 $\}, K=\operatorname{IntervalIntersection}[A, \operatorname{Interval}[\{a, b\}]] ; \mathbf{i}=\mathbf{1}$;

$\mathbf{B}=\mathbf{U n i o n}[\mathbf{K}, \operatorname{Interval}[\{\mathbf{b}, \mathbf{b}\}]] ;$

While $[\mathrm{B}[[\mathrm{i}]][[2]] \neq \operatorname{Max}[\mathrm{B}], \mathrm{i}=\mathrm{i}+1]$; Sum1 = 1;

$\mathbf{j}=\mathbf{1}$; While $[\mathbf{j} \leq \mathbf{i}$,

$\operatorname{Sum} 1=\operatorname{Sum} 1 * \operatorname{Exp}[\operatorname{Integrate}[\mathbf{p},\{\mathrm{x}, \mathrm{B}[[\mathbf{j}]][[1]], \mathrm{B}[[\mathbf{j}]][[2]]\}]] ; \mathbf{j}++;] ;$

$\mathrm{j}=1$; Sum2=1; While $[\mathrm{j}<\mathrm{i}$, Sum2 $=$ Sum2*

$\left.\operatorname{Exp}\left[\left(\left(p^{*} \log [2]\right) / . x \rightarrow B[[j]][[2]]\right) *(B[[j+1]][[1]]-B[[j]][[2]])\right] ; j++;\right] ;$

Print ["The Solution of the dynamic equation =", Sum1*Sum2]; ]

$\operatorname{In}[23]:=$ DynamicSolve $[1,0,10]$

Here the first component of the command DynamicSolve is the function $p$ of the dynamic equation $y^{\Delta}=p(t) y$, second is the initial point, and the third is the time at which we want to find the number of plants.

Another way of computing the number of plants without using the time scale integral is the following loop:

$\operatorname{In}[24]:=$ Clear $[\mathrm{yo}, \mathrm{f}, \mathrm{g}, \mathrm{a}, \mathrm{b}]$

$\operatorname{In}[25]:=y o=1 ; a=0 ; b=1$;

$\operatorname{In}[26]:=\operatorname{For}\left[\mathrm{i}=1, \mathrm{i}<6, \mathrm{~s}=\operatorname{DSolve}\left[\left\{\mathrm{y}^{\prime}[\mathrm{x}]=\mathrm{y}[\mathrm{x}], \mathrm{y}[\mathrm{a}]=\mathrm{yo}\right\}, \mathrm{y}[\mathrm{x}], \mathrm{x}\right] ;\right.$

$\mathrm{f}\left[\mathrm{x}_{-}\right]=\mathrm{y}[\mathrm{x}] / . \mathrm{s}[[1]] ; \mathrm{a}=\mathrm{a}+\mathbf{2} ; \mathrm{fd}=\operatorname{RSolve}[\{\mathrm{z}[\mathrm{x}+1]=\mathbf{z}[\mathrm{x}], \mathrm{z}[\mathrm{b}]=\mathrm{f}[\mathrm{a}-1]\}, \mathrm{z}[\mathrm{x}]$, $\left.\mathrm{x}] ; \mathrm{g}\left[\mathrm{x}_{-}\right]=\mathrm{z}[\mathrm{x}] / . \mathrm{fd}[[1]] ; \mathrm{b}=\mathrm{b}+2 ; \mathrm{yo}=\mathrm{g}[\mathrm{b}-1] ; \mathrm{i}++\right]$

$\operatorname{In}[27]:=$ yo

\section{Conclusion}

In this paper, we have given a very basic introduction to the time scales. Through a series of example, our goals were to present a variety of time scales, give the derivative, integral and dynamic equations on time scale. We compute some examples of each by Mathematica, and compare and contrast discrete and continuous calculus. But the symbolic computation in general for $\Delta$-derivative and the graphs of functions on time scales haven't done by Mathematica. It will be our future work. 


\section{References}

1. Agarwal, R.P.\& Bohner M., Basic calculus on time scale and some of its applications, Results Math. 35, (1-2) 3-22, (1999).

2. Agarwal, R.P., Bohner M., O'Regan, D.\& Peterson, A, Dynamic equations on time Scales: A survey, Journal of Computational and Applied Mathematics, Vol.141, (12), 1-26, (2002).

3. Aulbach B.\& Hilger, S., Linear dynamic process with in homogenous time scale in Nonlinear Dynamics and Quantum Dynamical Systems, Mathematical Research, Vol.59, Akademic Verlag Berlin, 9-20, (1999).

4. Bohner,M.\& Peterson,A., Dynamic Equations on Time Scales, Birkhäuser Boston, (2001).

5. Gray,J.W., Mastering Mathematica, Academic Press, (1997).

6. Hilger, S.: Analysis on measure chains-a unified approach to continuous and discrete calculus, Results Math. 18, 18-56, (1990).

7. Hilger, S., Differential and difference calculus-unified!, Nonlinear Anal. Theory Methods Appl. 30 (5), 2683-2694, (1997).

8. Wolfram,S., The Mathematica, Book, Cambrigde Univ. Press, (1996).

9. Yantir, A. Ufuktepe Ü., Basic Calculus on Time Scale with Mathematica, Lecture Notes in Computer Science, 2567, 821-827, (2003).

10. Yantir, A.Derivative and Integration on Time Scale with Mathematica, Challenging The Boundaries of Symbolic Computation: Proceeding of 5 th International Mathematica Symposium, 325-331., (2003). 